(2) Open Access Full Text Article

\title{
Sustained Clinical Benefit of Pyrotinib Combined with Capecitabine Rescue Therapy After Trastuzumab Resistance in HER2-Positive Advanced Gastric Cancer: A Case Report
}

\author{
Xin $\operatorname{Li}\left(\mathbb{D}^{1}{ }^{1} *\right.$ \\ Xiaoqiang $\mathrm{Gu}^{\mathrm{l}, *}$ \\ Jiahua $\mathrm{Xu}^{1, *}$ \\ Ling Chen $\left(\mathbb{D}^{2}\right)^{2, *}$ \\ Hongwei Li' \\ Dan Meng' \\ Haoran Bai' \\ Jinzu Yang' \\ Jianxin Qian'
}

'Department of Oncology, Longhua Hospital affiliated to Shanghai University of Traditional Chinese Medicine (TCM), Shanghai, 200032, People's Republic of China; ${ }^{2}$ Department of Oncology, Yueyang Hospital of Integrated

Traditional Chinese and Western

Medicine affiliated to Shanghai University of Traditional Chinese Medicine (TCM), Shanghai, 200437, People's Republic of China

*These authors contributed equally to this work

\begin{abstract}
Background: HER2-positive patients with advanced gastric cancer have a poor prognosis, and trastuzumab-resistant patients lack effective treatment.

Case Presentation: We report a 72-year-old male with HER2-positive gastric cancer. The patient had metastatic tumor during adjuvant chemotherapy after surgery, followed by second-line chemotherapy, and achieved a progression-free survival (PFS) of 4.5 months. Subsequent third-line chemotherapy treatment also failed. Fortunately, the patient had a significant tumor response and 8.5 months of PFS on trastuzumab combined with chemotherapy. After trastuzumab resistance, the patient was treated with programmed cell death protein-1 inhibitor combined with apatinib, which selectively inhibited VEGFR2, but the effect was not satisfactory. Finally, the patient was treated with capecitabine combined with pyrotinib, an irreversible TKI, acting on HER2. The tumor shrank significantly after this treatment.

Conclusion: The mechanism and countermeasures of trastuzumab resistance were discussed in this case. For patients with HER2-positive advanced gastric cancer, pyrotinib can achieve good results after trastuzumab resistance.
\end{abstract}

Keywords: HER2, gastric cancer, trastuzumab, pyrotinib

\section{Introduction}

Gastric cancer (GC) is one of the most common malignant tumors in the digestive tract, with high geographical and ethnic differences in incidence, among which the incidence in East Asia is the highest. ${ }^{1,2}$ However, East Asia also has the highest 5 -year survival rate for GC. The 5-year survival rate for GC patients in Japan is close to $70 \%{ }^{3}$ Thanks to the universal gastroscopy screening, the early diagnosis rate of GC patients in Japan is relatively high and the prognosis is good. ${ }^{4,5}$ Most Chinese patients with GC were found to be at an advanced stage, with a low 5-year survival rate. ${ }^{6}$ The main treatment methods for advanced GC include chemotherapy, molecular targeted therapy and immunotherapy.

In recent years, the use of targeted therapy against human epidermal growth factor receptor-2 (HER2) and immunotherapy against programmed cell death protein-1 (PD1) have made progress in the treatment of advanced GC. In addition, clinical studies targeting vascular endothelial growth factor receptor (VEGF) against GC have been
Correspondence: Jinzu Yang: Jianxin Qian Department of Oncology, Longhua Hospital affiliated to Shanghai University of Traditional Chinese Medicine (TCM) Shanghai, 200032, People's Republic of China

Email kingzuy@126.com;

jianxinqiang3@I63.com 
widely carried out. Phase III clinical study of apatinib, as an oral small molecule of VEGFR-2 tyrosine kinase inhibitor, in the treatment of advanced GC with failure of second-line chemotherapy showed that apatinib has an encouraging clinical effect. ${ }^{7}$ Based on this study, the China Food and Drug Administration approved apatinib as a third-line treatment for advanced GC.

ToGA study showed that trastuzumab combined with chemotherapy improved survival in HER2 overexpression of metastatic GC. ${ }^{8}$ Currently, there is no standard treatment for GC patients with trastuzumab resistance. ${ }^{9}$ Pyrotinib is a novel irreversible epidermal growth factor receptor (EGFR) and HER2 dual-tyrosine kinase inhibitor. A number of clinical studies have shown that pyrotinib alone or combined with capecitabine has excellent safety and curative effects in the treatment of HER2-positive metastatic breast cancer patients (including patients with trastuzumab resistance), ${ }^{10,11}$ hence CFDA approved pyrotinib plus capecitabine as the second-line standard-of-care for metastatic HER2-positive breast cancer in China. However, the efficacy of pyrotinib in trastuzumab-resistant GC patients remains unclear. Here, we report a case of advanced GC with HER2 positive that achieved favourable efficacy with pyrotinib after trastuzumab resistance.

\section{Case Presentation}

\section{Operation and First-Line Treatment}

A 72-year-old male patient underwent D2 radical gastrectomy in October 2017 due to elevated carbohydrate antigen 199 (CA199, $902 \mathrm{U} / \mathrm{mL}$ ) detected by routine examination and subsequently GC revealed by abdominal MRI and gastric adenocarcinoma by gastroscopy. Postoperative histopathological diagnosis was poorly differentiated from adenocarcinoma (Diffuse type) with HER2 positive (immunohistochemical), TNM staging (7th edition) was p-T4aN3aM0 (stage IIIC). After surgery, CA199 fell to $198 \mathrm{U} / \mathrm{mL}$ and the patient received six cycles of SOX (oxaliplatin $130 \mathrm{mg} / \mathrm{m}^{2}$, Tegafur Gimeracil Oteracil Potassium Capsule 60mg/d d1-14) regimens of adjuvant chemotherapy. In April 2018, Positron Emission Tomography-Computed Tomography (PET-CT) scan showed multiple larger lymph nodes in the right upper mesentery and abdominal aorta, the larger one was about $1.52 \times 1.03 \mathrm{~cm}$, and the maximum SUV was 3.84 , indicating lymph node metastasis. According to the consensus, this postoperative adjuvant chemotherapy should be considered as a first-line treatment. Figures 1-4 show the whole treatment process and the corresponding changes in PET-CT and computed tomography (CT) scan, as well as the changes in CA199 and carcinoembryonic antigen (CEA) levels.

\section{Second - and Third-Line Treatments}

In June 2018, CA199 (1239.0 U/mL) and CEA (18.7 ng/ $\mathrm{mL})$ were significantly elevated in this patient. We suggested that the patient should take trastuzumab combined with chemotherapy, but because trastuzumab was not covered by the medical insurance of Chinese residents at that time, the patient refused to use trastuzumab, so we gave the DP (docetaxel $75 \mathrm{mg} / \mathrm{m}^{2}$, cisplatin $75 \mathrm{mg} / \mathrm{m}^{2}$ ) chemotherapy to the patient. After two cycles of

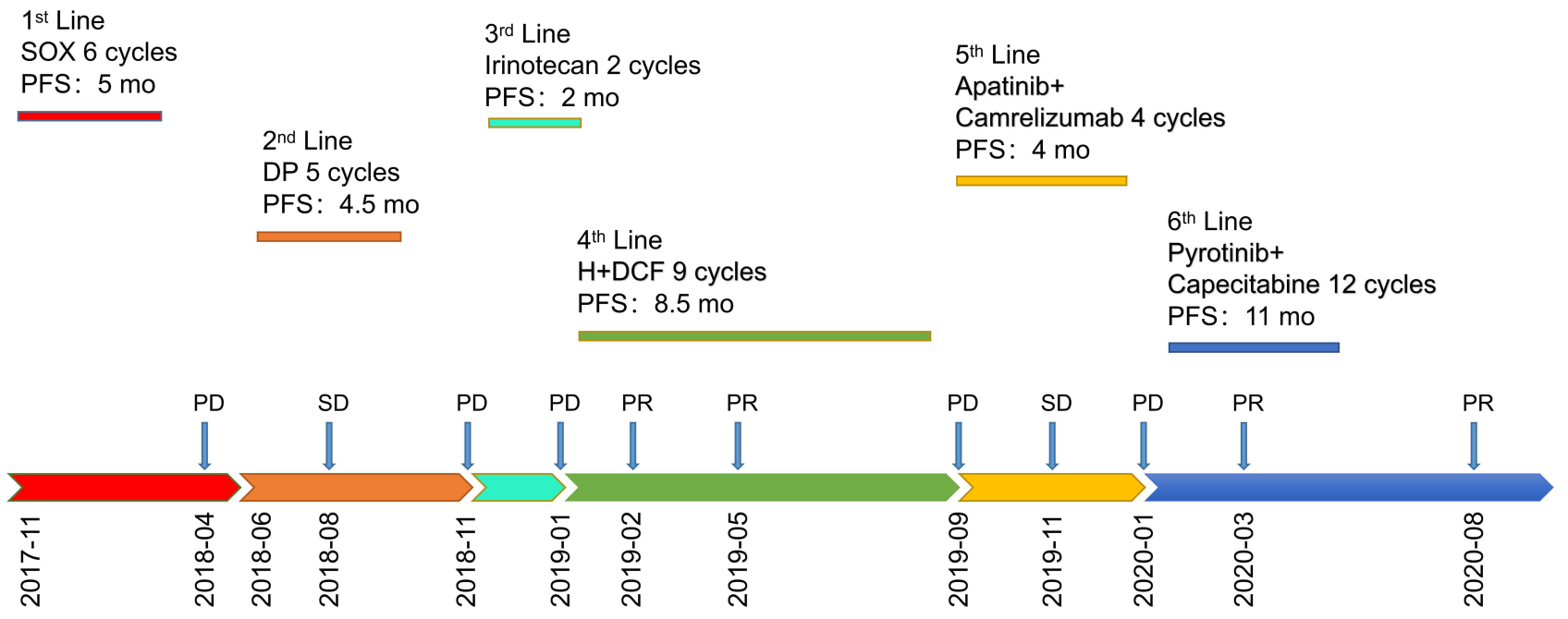

Figure I Disease development and treatment procedures.

Abbreviations: SOX, oxaliplatin/Tegafur Gimeracil Oteracil Potassium Capsule; DP, docetaxel/cisplatin; H, trastuzumab; DCF, docetaxel/cisplatin/5-fluorouracil; PFS, progression-free survival; PD, progressive disease; SD, Stable Disease; PR, partial response; mo, months. 


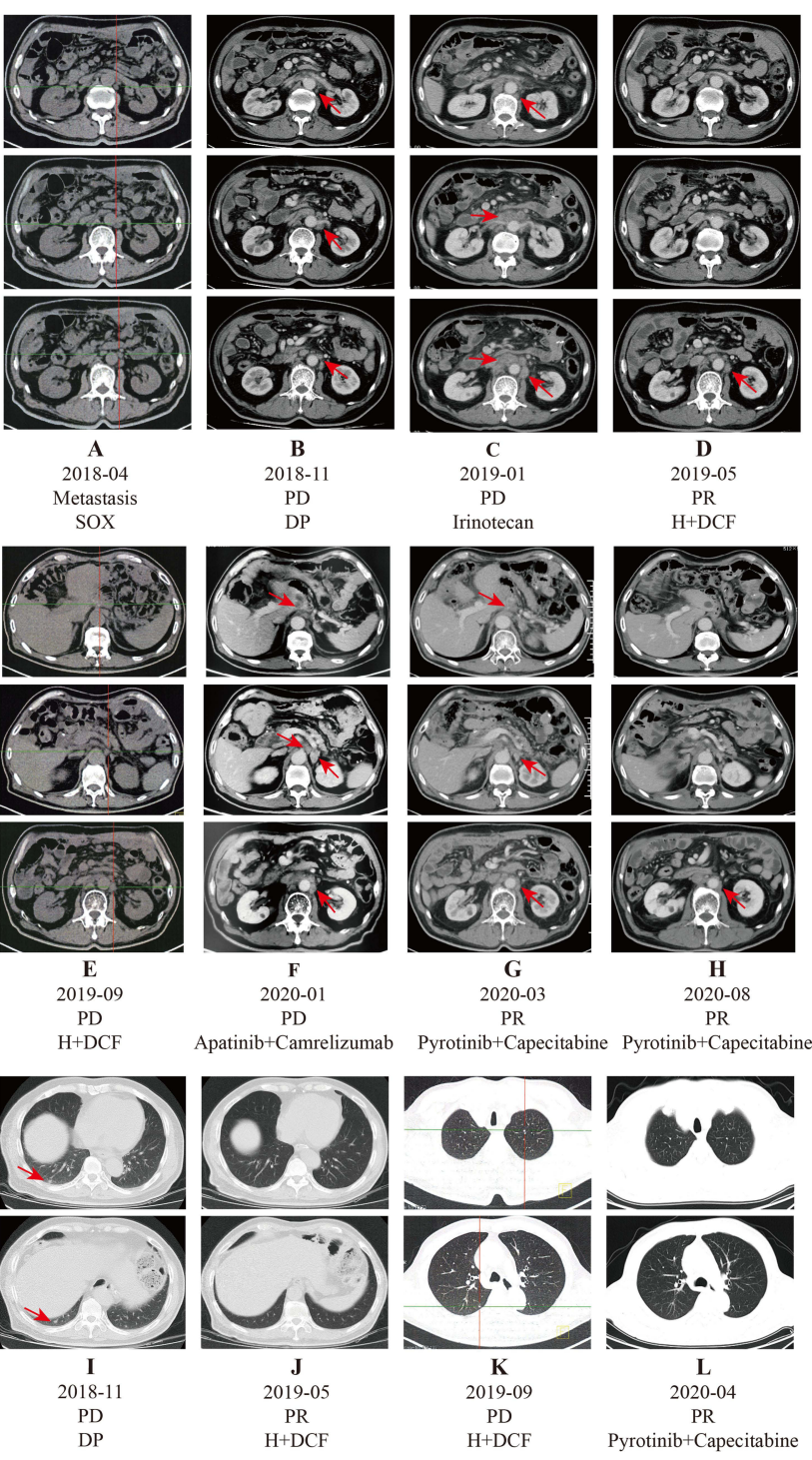

Figure 2 Whole body positron emission computed tomography (PET-CT) and computed tomography (CT) of chest and abdomen. (A) PET-CT showed multiple larger lymph nodes in the right upper mesentery and abdominal aorta after first-line chemotherapy (SOX). (B and I) CT demonstrates no significant changes in retroperitoneal lymph nodes and new lung metastases when DP failed. (C) CT demonstrated progressive disease (PD) in the retroperitoneal lymph nodes when irinotecan failed. ( $D$ and $\mathbf{J}$ ) CT scan indicated that the retroperitoneal lymph nodes were nearly disappeared and lung metastasis disappeared after four cycles of trastuzumab combined with chemotherapy (DCF) treatment. (E and K) PET-CT showed retroperitoneal newly metastatic lymph nodes and pulmonary newly metastatic nodules after trastuzumab combined with DCF. (F) After four cycles of apatinib combined with camrelizumab treatment, the size of retroperitoneal lymph nodes was significantly increased. (G, $\mathbf{H}$ and $\mathbf{L})$ After three cycles of pyrotinib combined with capecitabine treatment, retroperitoneal lymph nodes were smaller than before, pulmonary metastasis disappeared after four cycles, and CT scan in August 2020 showed that retroperitoneal lymph nodes almost disappeared.

chemotherapy, a CT scan of the patient's abdomen revealed a slight reduction in retroperitoneal lymph nodes. Until November 2018, new metastases were found on chest CT scan, accompanied by a significant increase in CA199 (8078.0 U/mL) and CEA (49.1 $\mathrm{ng} / \mathrm{mL})$.
Subsequently, he underwent two cycles of third-line chemotherapy with irinotecan $\left(250 \mathrm{mg} / \mathrm{m}^{2}\right)$. However, this regimen did not show therapeutic effect, accompanied by the continuous increase of CA199 $(16644.0 \mathrm{U} / \mathrm{mL})$ and CEA $(65.6 \mathrm{ng} / \mathrm{mL})$.

\section{The Fourth-Line Treatment of Trastuzumab Combined with Chemotherapy}

In January 2019, the patient developed back pain when a CT scan demonstrated progressive disease (PD) in the retroperitoneal lymph nodes. At this time, trastuzumab had been included in the medical insurance coverage of Chinese residents, so we treated the patient with trastuzumab $\left(6 \mathrm{mg} / \mathrm{kg}\right.$ ) combined with DCF (docetaxel $60 \mathrm{mg} / \mathrm{m}^{2}$, cisplatin $60 \mathrm{mg} / \mathrm{m}^{2}, 5$-fluorouracil $600 \mathrm{mg} / \mathrm{m}^{2} / \mathrm{d} \mathrm{d} 1-5$ ) regimen. After two cycles of therapy, the abdominal CT showed partial response (PR) of retroperitoneal lymph nodes, the back pain was significantly reduced. After four cycles of therapy, chest CT showed that pulmonary metastases disappeared, and abdominal CT showed PR of retroperitoneal lymph nodes. By August 2019, the patient received a total of 9 cycles of this regimen, during which CA199 was reduced to the lowest value of $206.0 \mathrm{U} / \mathrm{mL}$ and CEA to the lowest value of $9.3 \mathrm{ng} / \mathrm{mL}$. In September 2019, PET-CT showed new nodules in left lung and right lung, and multiple enlarged lymph nodes in retroperitoneal. The largest lymph node had a length of about $2.4 \mathrm{~cm}$, and the maximum SUV was 10.75 . At the same time, CA199 (3988.0 U/mL) and CEA $(30.2 \mathrm{ng} / \mathrm{mL})$ increased significantly. Comprehensive consideration, the patient's condition progress.

\section{The Fifth-Line Treatment of Apatinib Combined with Camrelizumab}

After the fourth-line treatment of GC, there is no standard treatment. We suggested genetic testing, and the patient refused. After comprehensive consideration, the patient was treated with anti-angiogenesis (apatinib 250mg/d) combined with anti-PD-1 inhibitor (camrelizumab, SHR$1210,200 \mathrm{mg}$ every 3 weeks). After two cycles of treatment, the abdominal CT scan showed no significant change in the size of the lesions. After that, we gave the patient two cycles of treatment again. In January 2020, CT scan showed that the retroperitoneal lymph nodes were enlarged, and CA199 (13725.0 U/mL) and CEA (48.3 $\mathrm{ng} / \mathrm{mL}$ ) were obviously increased. 


\section{CA199(U/mL)}

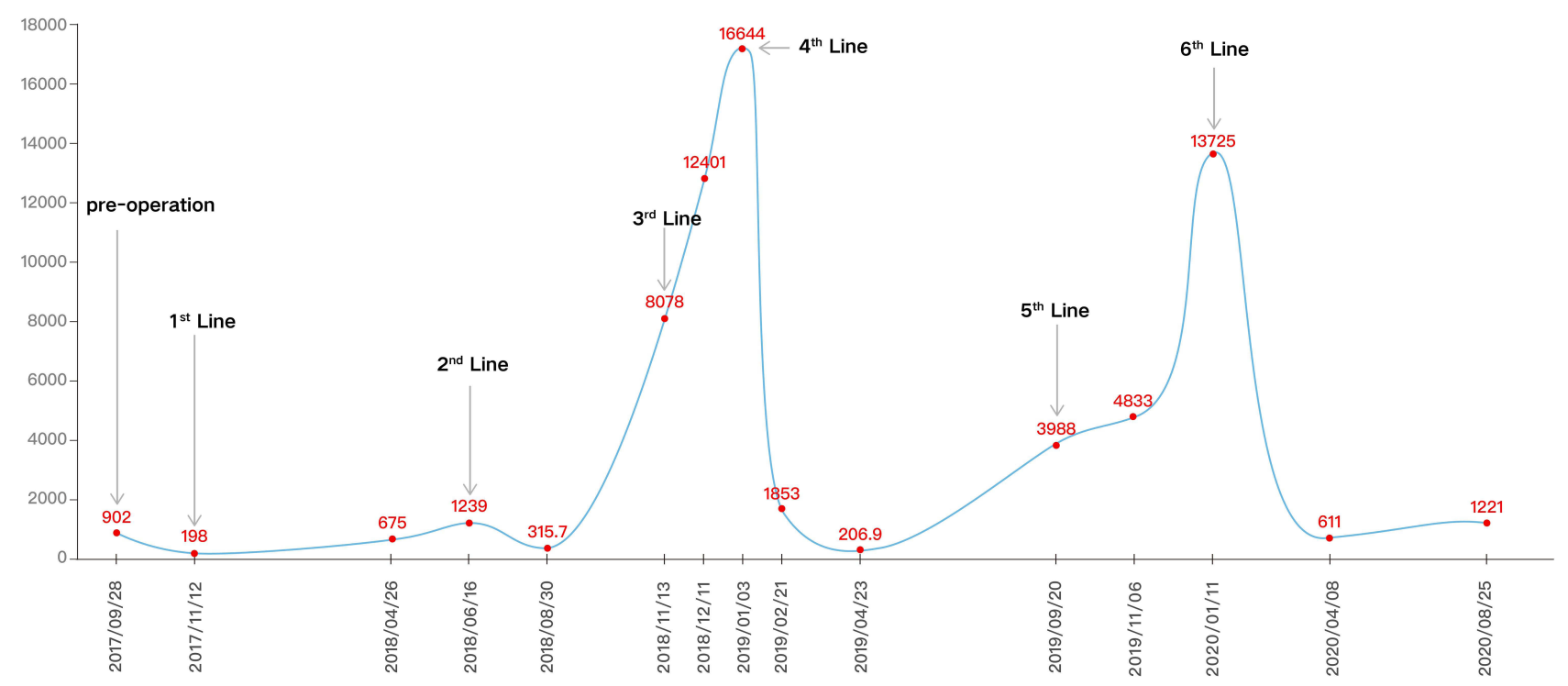

Figure 3 Change in carbohydrate antigen 199 (CA199) (U/mL) levels during entire treatment.

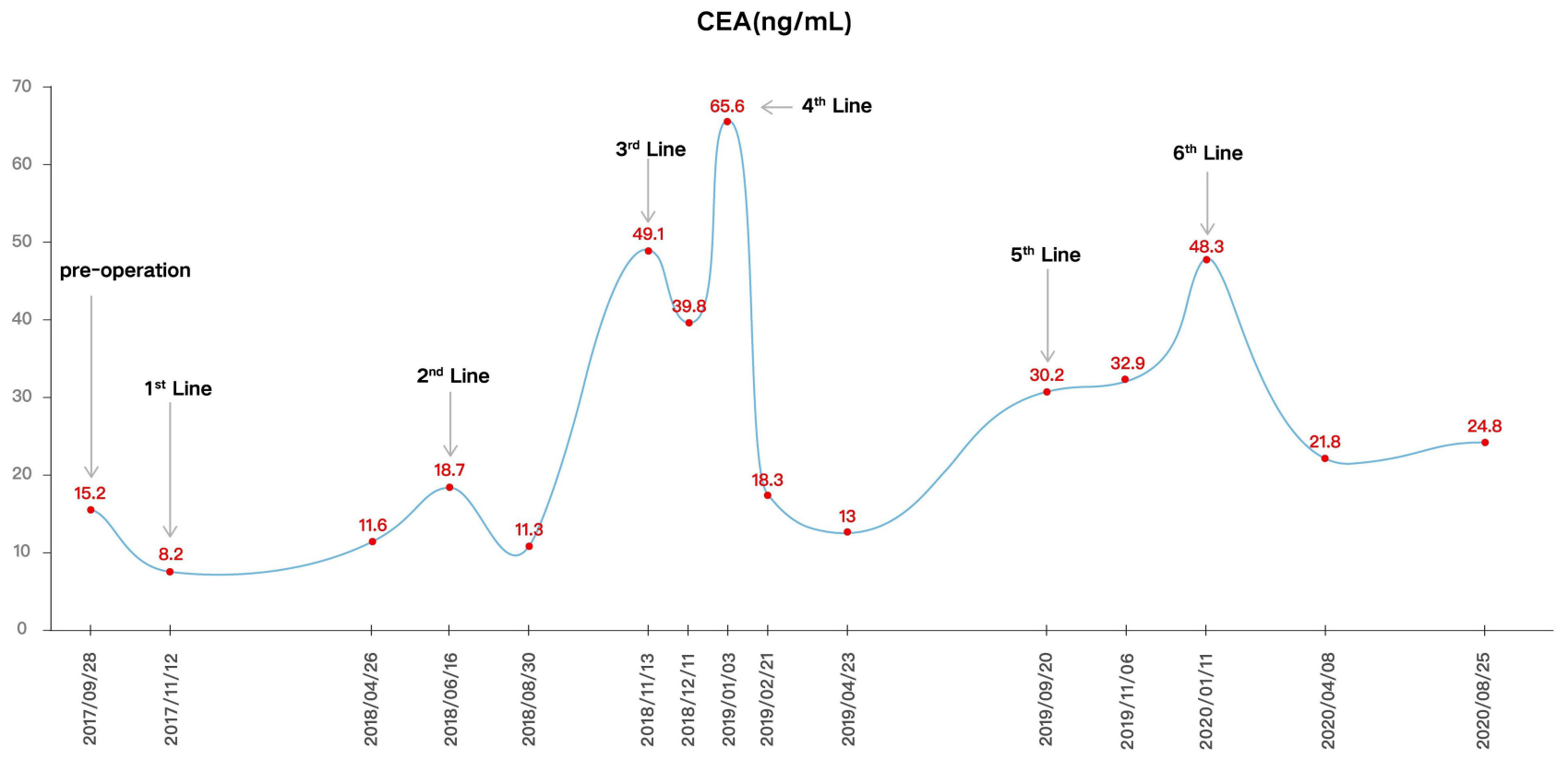

Figure 4 Change in carcinoembryonic antigen (CEA) $(\mathrm{ng} / \mathrm{mL})$ levels during entire treatment.

\section{The Sixth-Line Treatment of Pyrotinib Combined with Capecitabine}

After the patient's disease progresses, we recommend genetic testing again. Peripheral blood tests were performed because the patient's pathological tissue was difficult to obtain. Next-generation sequencing (NGS) (543 genes; GeneCast Biotechnology Co. Ltd., Beijing, China) was performed. NGS results showed that the patient had an increased copy number of HER2 gene, no HER2 gene mutation, and a negative expression of programmed cell death ligand 1 (PD-L1). Detailed results are listed in Table 1 . We conducted a multidisciplinary treatment discussion on the patient's condition in our hospital, and found that there is no standard treatment plan for the six-line 
Table I Genetic Test Results of Patients

\begin{tabular}{|l|l|l|l|l|}
\hline GENES & Variations & Abundance & GCN & Methodology \\
\hline HER2 & Increased GCN & & 15.62 & NGS \\
CDHI & p.E497K & $2.07 \%$ & & \\
DNMT3A & p.V636M & $0.83 \%$ & & \\
KMT2C & p.1707T & $0.81 \%$ & 3.13 \\
TP53 & p.C242Y & $1.83 \%$ & 1.43 \\
CDK4 & Decreased GCN & & & \\
PTEN & Decreased GCN & $1.27 / M b$ & & \\
TMB & Low & & \\
MSI & MSS/MSI-L & & \\
KRAS & Negative & & \\
\hline
\end{tabular}

Abbreviations: HER2, human epidermal growth factor receptor-2; CDHI, cadherin I; DNMT3A, DNA methyltransferase 3A; KMT2C, Lysine methyltransferase 2C; TP53, tumor protein p53; CDK4, cyclin-dependent kinase 4; PTEN, phosphatase and tensin homolog; TMB, tumor mutational burden; MSI, microsatellite instability; MSS, microsatellite stability; MSI-L, microsatellite instability low; KRAS, Kirsten rat sarcoma viral oncogene; GCN, gene copy number; NGS, next generation sequencing.

treatment of advanced GC, and no relevant clinical trial has been carried out in our hospital. According to the NGS results, the copy number of the HER2 gene was significantly increased in this patient. Pyrotinib, a novel HER2 inhibitor, has been approved in combination with capecitabine in China for patients with relapsed or metastatic breast cancer who had previously received trastuzumab. We hypothesized that pyrotinib combined with capecitabine might be effective in this patient. Therefore, with the patient's informed consent, the patient was administered pyrotinib (400mg/d) plus capecitabine $(1000 \mathrm{mg} / \mathrm{m} 2$, bid, d1-14, every 3 weeks) treatment in January 2020. After three cycles, abdominal CT scans showed PR in the retroperitoneal lymph node. After four cycles, chest CT scans showed that pulmonary nodules disappeared. At the same time, CA199 and CEA decreased steadily. In August 2020, abdominal CT still showed PR in retroperitoneal lymph nodes. The patient tolerated treatment with pyrotinib well. No adverse events were observed. Until the submission of the case draft, the patient has survived for over 30 months since postoperative recurrence and now continues to receive the combination treatment of pyrotinib plus capecitabine, and the PFS is over 8.5 months.

\section{Discussion}

HER2-positive GC is a unique subtype of GC, which requires different diagnosis and treatment strategies from HER2 negative GC. A retrospective study showed that HER2-positive expression was associated with older age, male, histological Lauren classification of intestinal type, and tumor located in the upper $1 / 3$ of the stomach. ${ }^{12}$ Studies have also shown that HER2 overexpression is associated with poor prognosis in patients with GC. HER2 negative intestinal type GC has the best survival outcome, while HER2-positive diffuse GC has the worst outcome. $^{13}$

Trastuzumab is presently the only drug with definite clinical evidence and proven efficacy in treating HER2positive GC. Many clinical studies have confirmed that chemotherapy combined with trastuzumab can significantly improve ORR and OS in patients with advanced HER2-positive GC. ${ }^{8,14-16}$ The case we reported was not treated with trastuzumab at first. After continuous multiline chemotherapy, the patient's condition did not reach remission. After receiving trastuzumab therapy, the clinical symptoms were incredibly improved and 8.5 months of PFS were obtained. Although the subsequent combination of apatinib and camrelizumab achieved only 4 months of PFS, the follow-up pyrotinib treatment has again achieved 8.5 months of PFS. The continued effectiveness of anti-HER2 therapy suggests that HER2 pathway is a key factor in the progression of cancer in this patient.

After trastuzumab resistance, there was no standard treatment regimen. With the rapid development of immune checkpoint inhibitors in tumors, immunotherapy for GC has also been rapidly developed. Nivolumab has obvious survival benefits in the treatment of patients with advanced GC or gastroesophageal junction cancer who have previously received two or more chemotherapy regimens. ${ }^{17}$ Keynote-059 study showed that the objective remission rate of GC patients receiving pembrolizumab second-line or multi-line treatment was $11.6 \%{ }^{18}$ Studies have shown that Camrelizumab has an encouraging effect in patients with advanced GC after chemotherapy in China. ${ }^{19}$ Moreover, a number of studies have shown that anti- 
angiogenesis therapy combined with immunotherapy can reshape the tumor immunosuppressive microenvironment and improve the effect of anti-tumor therapy. ${ }^{20-23}$ Therefore, we treated the patient with apatinib combined with Camrelizumab, and the patient received 4 months of PFS. The patient initially refused the genetic test, so we could not predict the efficacy of the immunotherapy, so giving the immunotherapy to the patient did not yield surprising results. At present, a series of advances have been made in immunotherapy for gastric cancer, but the dominant population who can benefit from immunotherapy is still not clear, so further studies are needed to explore effective biomarkers.

Genetic testing of the patient prior to six-line treatment showed HER2 amplification, suggesting that the patient's condition was closely related to the HER2 pathway. So, our therapy is still focused on anti-HER2 therapy. At present, the mechanism of trastuzumab resistance is not clear. Trastuzumab is a recombinant humanized monoclonal antibody that specifically acts on the extracellular domain of HER2 and only acts on the HER2 pathway. Pyrotinib permanently binds to ATP binding sites in the intracellular kinase regions of HER1, HER2 and HER4, completely blocking the downstream pathway activated by homodimers or heterodimers of HER family on the tumor cell membrane. ${ }^{24}$ Based on the difference of action mechanism, the encouraging results of pyrotinib combined with capecitabine in HER2-positive advanced breast cancer, and the favorable results of pyrotinib in preclinical and clinical studies in GC, ${ }^{25,26}$ we innovatively treated this patient with pyrotinib plus capecitabine, which has benefited the patient's survival to this day. In view of the successful experience of this case and the mechanism of pyrotinib, pyrotinib is suitable for the rescue treatment of trastuzumab resistance. Of course, large clinical studies are needed to confirm this. We were delighted to find that a Phase I study of pyrotinib combined with SHR6390 in the treatment of HER2-positive GC (NCT03480256) is currently underway and we look forward to the experimental results of this study.

\section{Conclusions}

We report a case of advanced GC after trastuzumab treatment resistance. Subsequently, pyrotinib was used for treatment, and achieved surprising clinical efficacy. Clinical trials of pyrotinib for HER2-positive advanced $\mathrm{GC}$ are currently underway.

\section{Data Sharing Statement}

The data used in the case are available from the corresponding author on reasonable request.

\section{Ethics Statement}

The written informed consent of the patient was obtained for the publication of this case report and any identifying information and images. The protocol was approved by the Medical Ethics Committee of Longhua Hospital Affiliated to Shanghai University of Chinese Medicine in accordance with the requirements of Chinese laws and regulations and the ethical requirements of the Helsinki Declaration.

\section{Funding}

This work was partially supported by Genecast Cancer Research Project of Beijing CSCO Clinical Cancer Research Foundation (Y2019Genecast-074).

\section{Disclosure}

The authors declare that they have no conflicts of interest.

\section{References}

1. Li TT, Liu H, Yu J, Shi GY, Zhao LY, Li GX. Prognostic and predictive blood biomarkers in gastric cancer and the potential application of circulating tumor cells. World J Gastroenterol. 2018;24 (21):2236-2246. doi:10.3748/wjg.v24.i21.2236

2. Schumacher SE, Shim BY, Corso G, et al. Somatic copy number alterations in gastric adenocarcinomas among Asian and Western patients. PLoS One. 2017;12(4):e0176045. doi:10.1371/journal. pone. 0176045

3. Allemani C, Matsuda T, Di Carlo V, et al. Global surveillance of trends in cancer survival 2000-14 (CONCORD-3): analysis of individual records for 37513025 patients diagnosed with one of 18 cancers from 322 population-based registries in 71 countries. Lancet. 2018;391(10125):1023-1075.

4. Sumiyama K. Past and current trends in endoscopic diagnosis for early stage gastric cancer in Japan. Gastric Cancer. 2017;20(Suppl 1):20-27. doi:10.1007/s10120-016-0659-4

5. Katai H, Ishikawa T, Akazawa K, et al. Five-year survival analysis of surgically resected gastric cancer cases in Japan: a retrospective analysis of more than 100,000 patients from the nationwide registry of the Japanese gastric cancer association (2001-2007). Gastric Cancer. 2018;21(1):144-154. doi:10.1007/s10120-017-0716-7

6. Chen W, Zheng R, Baade PD, et al. Cancer statistics in China, 2015. CA Cancer J Clin. 2016;66(2):115-132. doi:10.3322/caac.21338

7. Li J, Qin S, Xu J, et al. Randomized, double-blind, placebo-controlled phase iii trial of apatinib in patients with chemotherapy-refractory advanced or metastatic adenocarcinoma of the stomach or gastroesophageal junction. J Clin Oncol. 2016;34(13):1448-1454. doi:10.1200/ JCO.2015.63.5995

8. Bang YJ, Van Cutsem E, Feyereislova A, et al. Trastuzumab in combination with chemotherapy versus chemotherapy alone for treatment of HER2-positive advanced gastric or gastro-oesophageal junction cancer (ToGA): a Phase 3, open-label, randomised controlled trial. Lancet. 2010;376(9742):687-697. doi:10.1016/S0140-6736(10)61121$\mathrm{X}$ 
9. Jomrich G, Schoppmann SF. Targeting HER 2 and angiogenesis in gastric cancer. Expert Rev Anticancer Ther. 2016;16(1):111-122. doi:10.1586/14737140.2016.1121110

10. Ma F, Li Q, Chen S, et al. Phase I study and biomarker analysis of pyrotinib, a novel irreversible pan-erbb receptor tyrosine kinase inhibitor, in patients with human epidermal growth factor receptor 2-positive metastatic breast cancer. J Clin Oncol. 2017;35 (27):3105-3112. doi:10.1200/JCO.2016.69.6179

11. Ma F, Ouyang Q, Li W, et al. Pyrotinib or lapatinib combined with capecitabine in HER2-positive metastatic breast cancer with prior taxanes, anthracyclines, and/or trastuzumab: a randomized, Phase II study. J Clin Oncol. 2019;37(29):2610-2619. doi:10.1200/ JCO.19.00108

12. Qiu M, Zhou Y, Zhang X, et al. Lauren classification combined with HER2 status is a better prognostic factor in Chinese gastric cancer patients. BMC Cancer. 2014;14(1):823. doi:10.1186/1471-2407-14823

13. Lei YY, Huang JY, Zhao QR, et al. The clinicopathological parameters and prognostic significance of HER2 expression in gastric cancer patients: a meta-analysis of literature. World J Surg Oncol. 2017;15(1):68. doi:10.1186/s12957-017-1132-5

14. Qiu MZ, Li Q, Wang ZQ, et al. HER2-positive patients receiving trastuzumab treatment have a comparable prognosis with HER2-negative advanced gastric cancer patients: a prospective cohort observation. Int $J$ Cancer. 2014;134(10):2468-2477. doi:10.1002/ijc.28559

15. Gong J, Liu T, Fan Q, et al. Optimal regimen of trastuzumab in combination with oxaliplatin/ capecitabine in first-line treatment of HER2-positive advanced gastric cancer (CGOG1001): a multicenter, phase II trial. BMC Cancer. 2016;16(1):68. doi:10.1186/s12885-0162092-9

16. Ryu MH, Yoo C, Kim JG, et al. Multicenter phase II study of trastuzumab in combination with capecitabine and oxaliplatin for advanced gastric cancer. Eur J Cancer. 2015;51(4):482-488. doi:10.1016/j.ejca.2014.12.015

17. Kang YK, Boku N, Satoh T, et al. Nivolumab in patients with advanced gastric or gastro-oesophageal junction cancer refractory to, or intolerant of, at least two previous chemotherapy regimens (ONO-4538-12, ATTRACTION-2): a randomised, double-blind, placebo-controlled, phase 3 trial. Lancet. 2017;390 (10111):2461-2471. doi:10.1016/S0140-6736(17)31827-5
18. Fuchs CS, Doi T, Jang RW, et al. Safety and efficacy of pembrolizumab monotherapy in patients with previously treated advanced gastric and gastroesophageal junction cancer: Phase 2 clinical KEYNOTE-059 Trial. JAMA Oncol. 2018;4(5):e180013. doi:10.1001/jamaoncol.2018.0013

19. Huang J, Mo H, Zhang W, et al. Promising efficacy of SHR-1210, a novel anti-programmed cell death 1 antibody, in patients with advanced gastric and gastroesophageal junction cancer in China. Cancer. 2019;125(5):742-749. doi:10.1002/cncr.31855

20. Huang Y, Yuan J, Righi E, et al. Vascular normalizing doses of antiangiogenic treatment reprogram the immunosuppressive tumor microenvironment and enhance immunotherapy. Proc Natl Acad Sci US A. 2012;109(43):17561-17566. doi:10.1073/pnas.1215397109

21. Huang Y, Goel S, Duda DG, Fukumura D, Jain RK. Vascular normalization as an emerging strategy to enhance cancer immunotherapy. Cancer Res. 2013;73(10):2943-2948. doi:10.1158/0008-5472.CAN$12-4354$

22. Zhao S, Ren S, Jiang T, et al. Low-dose apatinib optimizes tumor microenvironment and potentiates antitumor effect of PD-1/PD-L1 blockade in lung cancer. Cancer Immunol Res. 2019;7(4):630-643. doi:10.1158/2326-6066.CIR-17-0640

23. Xu J, Zhang Y, Jia R, et al. Anti-PD-1 antibody SHR-1210 combined with apatinib for advanced hepatocellular carcinoma, gastric, or esophagogastric junction cancer: an open-label, dose escalation and expansion Study. Clin Cancer Res. 2019;25(2):515-523. doi:10.1158/1078-0432.CCR-18-2484

24. Li X, Yang C, Wan H, et al. Discovery and development of pyrotinib: a novel irreversible EGFR/HER2 dual tyrosine kinase inhibitor with favorable safety profiles for the treatment of breast cancer. Eur J Pharm Sci. 2017;110:51-61. doi:10.1016/j.ejps.2017.01.021

25. Huang T, Luo X, Wu B, et al. Pyrotinib enhances the radiosensitivity of HER2-overexpressing gastric and breast cancer cells. Oncol Rep. 2020;44(6):2634-2644. doi:10.3892/or.2020.7820

26. Yin Y, Yang H, Liu Z, et al. Studies on the safety and efficacy of pyrotinib in the treatment of HER2- positive advanced solid tumors excluding breast cancer. Cancer Manag Res. 2020;12:13479-13487. doi:10.2147/CMAR.S281765
OncoTargets and Therapy

\section{Publish your work in this journal}

OncoTargets and Therapy is an international, peer-reviewed, open access journal focusing on the pathological basis of all cancers, potential targets for therapy and treatment protocols employed to improve the management of cancer patients. The journal also focuses on the impact of management programs and new therapeutic

Submit your manuscript here: https://www.dovepress.com/oncotargets-and-therapy-journal
Dovepress

agents and protocols on patient perspectives such as quality of life, adherence and satisfaction. The manuscript management system is completely online and includes a very quick and fair peer-review system, which is all easy to use. Visit http://www.dovepress.com/ testimonials.php to read real quotes from published authors. 